\title{
INFRARED MEASUREMENTS OF STELLAR MAGNETIC FIELDS
}

\author{
STEVEN H. SAAR \\ Center for Astrophysics, 60 Garden Street, Cambridge, MA 02138, U.S.A.
}

\begin{abstract}
I review the advantages, techniques, and results of measurement of magnetic fields on cool stars in the infrared (IR). These measurements have generated several important results, including the following: the first data on the magnetic parameters of dMe and RS CVn variables; evidence for field strength confinement by photospheric gas pressure; support for the correlation between magnetic flux and rotation, with possible saturation at high rotation rates; indications of horizontal and/or vertical magnetic field structure; and evidence of spatial variations in $B$ over a stellar surface. I discuss these results in detail, and suggest future directions for IR magnetic field research.
\end{abstract}

Key words: infrared: stars - line: formation - stars: activity - stars: magnetic fields

\section{Introduction}

Welcome to probably one of the shorter reviews in this volume. The main reason for its brevity is that infrared measurements of stellar magnetic fields have only been made since 1982 and with only one instrument: the NOAO 4-m Fourier Transform Spectrometer (Hall et al. 1979). Until very recently, this was the only instrument in the world capable of the high resolution $(\lambda / \Delta \lambda \gtrsim 40,000)$, low noise (S/N $z$ $50)$ spectra needed to measure magnetic parameters from unpolarized absorption lines. The exposure times needed were long, however $\left(\approx 6\right.$ hours for $\left.\mathrm{m}_{K}=5\right)$, and consequently only a handful of stars have been studied to date. With the advent of cryogenic echelles and efficient IR arrays, there should be an explosion of new data in the near future. Thus, this review can be considered a summary of the "first generation" IR stellar field detections - few in number, but important in shaping our understanding of magnetic fields on solar-like stars.

Infrared observations to detect stellar magnetic fields on solar-like stars have several advantages. The most obvious of these is that the ratio of the Zeeman splitting to the Doppler width, $\Delta \lambda_{B} / \Delta \lambda_{D}$, is proportional to $\lambda$, making detection of typical stellar field strengths ( $B \sim 1 \mathrm{kG}$ or more) considerably easier in the IR. Comparison with the half widths of typical moderate-strength lines $(\sim 3 \mathrm{~km}$ $\mathrm{s}^{-1}$ ) makes this clear. The Zeeman width splitting (in $\mathrm{km} \mathrm{s}^{-1}$ ) is $v_{B}=1.7 \times$ $10^{-4} g_{\mathrm{eff}} \lambda B$ (where $\lambda$ is in $\AA$, and $B$ in $\mathrm{kG}$ ), and so the improvement in detectability, in proportion to $\lambda$, changes a slight (and difficult to interpret) line broadening at optical wavelengths (e.g., $v_{B}=2.6 \mathrm{~km} \mathrm{~s}^{-1}$ for $g_{\mathrm{eff}}=2.5$ and $B=1 \mathrm{kG}$ at $0.6 \mu \mathrm{m}$ ), to partial or full line splitting in the IR $\left(v_{B}=9.4 \mathrm{~km} \mathrm{~s}^{-1}\right.$ for the same line at $2.2 \mu \mathrm{m})$. This increased sensitivity is particularly helpful in low-gravity RS CVn systems, where attempts at optical field measurements have mostly failed, probably because of low $B$ values (Marcy and Bruning 1984; Saar 1990).

Other advantages of the IR include lower line density, so that blends are less likely to confuse a measurement, and that, for cooler stars at least, the near IR is close to the stellar flux maximum. Both of these are important for $\mathbf{M}$ dwarfs $\left(\mathrm{T}_{\text {eff }} \leq 3,750 \mathrm{~K}\right)$, whose optical spectra are riddled with molecular bands. Since the difference between umbral and plage/network continuum levels is considerably lower in the IR, IR spectra also hold the potential for disentangling the various 

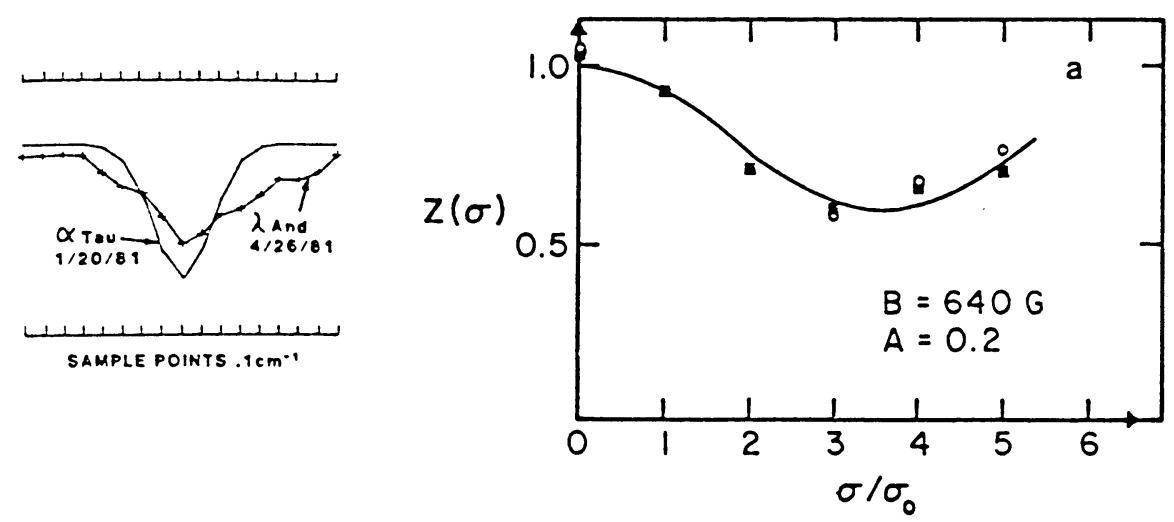

Fig. 1. Left: Comparison of the $g_{\text {eff }}=3,1.56 \mu \mathrm{m}$ line in $\lambda$ And and $\alpha$ Tau; a Fourier ratio analysis yields $B=1,290 \mathrm{G}$ and $f=0.48$ (from Giampapa et al. 1983). Right: Fourier ratio $\mathrm{z}(\sigma) v s$. frequency, $\sigma$, of two $\mathrm{Fe} I$ lines (o), again from $\lambda$ And, but at a different epoch (from Gondoin et al. 1985). A fit yielded $B \approx 600 \mathrm{G}$ and $f \approx 0.20$ (solid).

different types of magnetic regions expected on stars (optical spectra, due to the high contrast, can only detect the brighter magnetic regions). Finally, IR spectra offer new windows on the vertical structure of magnetic fields in stellar atmospheres, as the $1.6 \mu \mathrm{m}$ opacity minimum permits $B$ measurements deep into the photosphere, and the $12 \mu \mathrm{m} \mathrm{Mg}$ I lines sample the upper photosphere.

\section{A Short History of IR Stellar Magnetic Field Research}

Robinson, Worden, and Harvey (1980) made the first unambiguous detection of fields on a cool non-solar star ( $\xi$ Boo A), using the Fourier ratio method (Robinson 1980). This method assumes the lines are optically thin and treats a high- $g_{\text {eff }}$ line as a convolution of three $g_{\text {eff }}=0$ features (approximated by low- $g_{\text {eff }}$ lines in the same spectrum). The ratio of the Fourier transforms of the lines then yields a cosine function whose amplitude is related to the fraction, $f$, of the stellar surface covered by fields, and whose period is proportional to the mean field strength, $B$, in these regions (see also Gray 1984). Three years later, Giampapa, Golub, and Worden (1983) made the first sortie into the IR, studying the popular $1.56 \mu \mathrm{m}$ $g_{\text {eff }}=3.0 \mathrm{Fe}$ I line in the long period RS CVn star $\lambda$ And (Fig. 1). Although they obtained several spectra (Giampapa and Worden 1982), only one showed clear Zeeman broadening in several lines (perhaps due to lower $S / N$ in the other spectra). In a slight modification of the Robinson method, they took the same line from a different, low-activity star $\left(\alpha\right.$ Tau) as their approximate $g_{\text {eff }}=0$ line. From the one spectrum with a clear signal, they obtained $B=1,290 \mathrm{G}$ and $f=0.48$ - the first detection of magnetic fields on a giant star.

Two years later, Gondoin, Giampapa, and Bookbinder (1985) returned to $\lambda$ And for further study (see Fig. 1). Perhaps because it becomes more strongly blended 
in cooler stars, and is thus difficult to use in a standard Fourier ratio analysis, the $g_{\text {eff }} 3.0$ line was not used in this work. Instead, Gondoin et al. studied pairs of high $\left(g_{\text {eff }}>1.5\right)$ and low $g_{\text {eff }}$ lines with the basic Robinson (1980) method, and obtained a marginal detection for $B=600 \mathrm{G}$ and $f=0.20$. They attributed the difference between these values and those of Giampapa et al. (1983) to changing mixtures of spot (higher $B$ ) and plage (lower $B$ ) on the stellar surface at the two epochs. This is not unreasonable, as $\lambda$ And is known to have large spotted regions on its surface, showing photometric amplitudes, $\Delta \mathrm{V}$, up to 0.28 (Strassmeier et al. 1988). If their interpretation is correct, it represents the first evidence for differing magnitudes of $B$ in spot and plage regions on stars. Gondoin et al. (1985) also observed $\xi$ Boo A and $61 \mathrm{UMa}$, detecting no clear evidence for fields on either. The null result for $\xi$ Boo A is surprising, as most other measurements of the star have yielded positive detections. A list of further useful lines in the $1.6 \mu \mathrm{m}$ region has been tabulated by Solanki, Biémont, and Mürset (1990).

Sun, Giampapa, and Worden (1987) explored the possible uses of IR lines in distinguishing between stellar spot and plage fields. Using $1.6 \mu \mathrm{m}$ solar spectra (from the NSO McMath FTS), they combined varying flux-weighted fractions of umbral, plage, and quiet profiles, and analyzed the results as if they were stellar exposures. They found that spot umbrae dominate the derived $f$ if $f_{\text {spot }} \geq 0.10$.

Meanwhile, others pushed further into the IR. Saar and Linsky (1985) noted a Ti I multiplet at $2.2 \mu \mathrm{m}$ seen in umbral spectra (e.g., Hall 1973) with a helpfully large range of $g_{\text {eff }}$ values (from $\sim 1.0$ to $\sim 2.5$, with the latter, at $4,480 \mathrm{~cm}^{-1}$, having a Zeeman sensitivity $g_{\mathrm{eff}} \lambda$ equal to that of the $1.56 \mu \mathrm{m}$ line). They reasoned that this multiplet might be useful in studying fields on cool $\mathrm{K}$ and $\mathrm{M}$ stars, which are difficult to study in the optical due to blends. Their efforts were rewarded when only a six hour exposure of the active dM3.5e flare star AD Leo showed evidence for clear splitting of Zeeman components in several Ti I lines (Fig. 2). Using the simple Unno (1956) radiative transfer solution for Stokes $I$, and convolving the result with appropriate rotation and turbulent broadening functions, Saar and Linsky (1985) derived an average of $B \approx 3,800 \mathrm{G}$ and $f \approx 0.73$ from five Ti I lines. This detection was the first for an $\mathrm{M}$ dwarf, and the strongest field discovered up to that point on a cool star.

Continuing along these lines, Saar, Linsky, and Giampapa (1987) studied a number of late $\mathrm{K}$ and $\mathrm{M}$ dwarfs using the Ti I lines, plus two intermediate $g_{\text {eff }}(1.0$ and 1.33) $\mathrm{Na} I$ lines in the same bandpass. Only one of the six dM stars they studied showed a field (a marginal detection of the weak flare star GL 229), contrary to expectations of large $f$ values on dM stars (Giampapa 1985; Fig. 3). On the other hand, all dMe stars showed strong $(B>2 \mathrm{kG})$, widespread $(f>0.5)$ fields, consistent with their high activity and flaring rates. The field strengths, though smaller than some predictions (Mullan 1985), followed the trend indicated by $G$ and $\mathrm{K}$ dwarfs, namely larger $B$ values with decreasing $\mathrm{T}_{\text {eff }}$ and increasing gas pressure, consistent with $B \propto P_{\text {gas }}^{0.5}$. The IR data also confirmed and extended the correlation between $f$ or $f B$ and rotation seen in optical magnetic data (Saar and Linsky 1986). No measurable field was detected on the old K5 dwarf 61 Cyg A, suggesting previous optical detections may have been misled by subtle blends.

Jennings et al. (1986) used a cryogenic postdisperser and a BIB detector with 


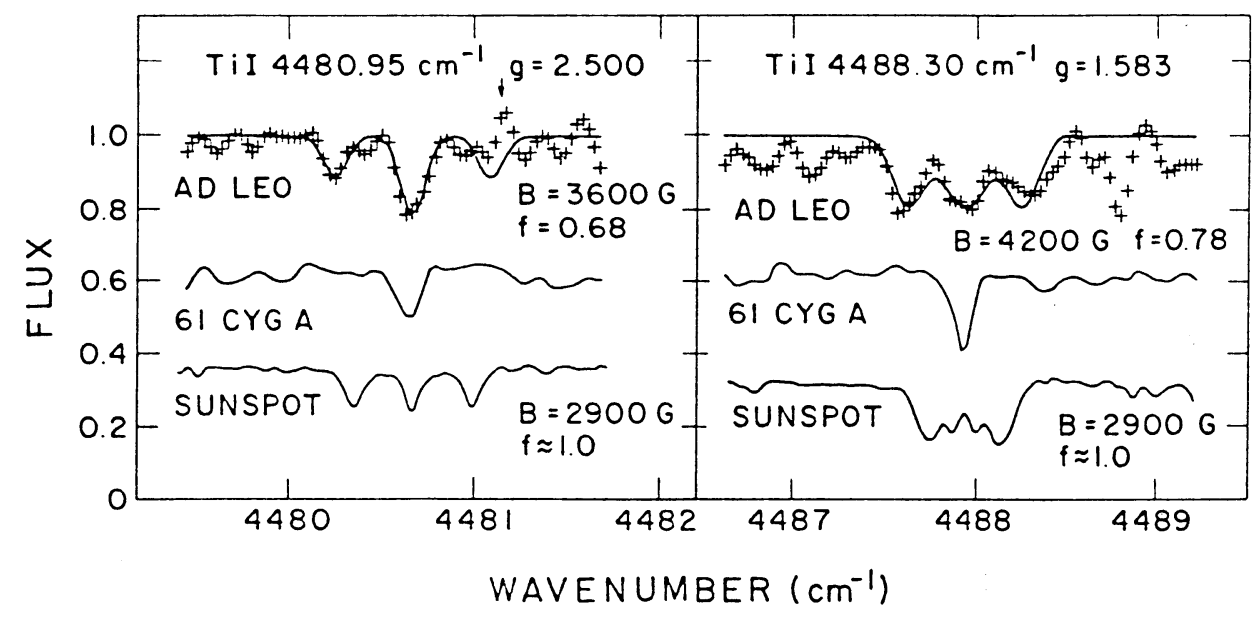

Fig. 2. Comparison of apodized 4-m FTS spectra of AD Leo (crosses), 61 Cyg A (an inactive $\mathrm{K} 5 \mathrm{~V}$ ) and a umbra/photosphere ratio spectrum from Hall (1973). Fits to AD Leo for the indicated $f$ and $B$ values are also shown (solid). Areas of enhanced noise due to telluric line removal are marked by arrows (from Saar and Linsky 1985).

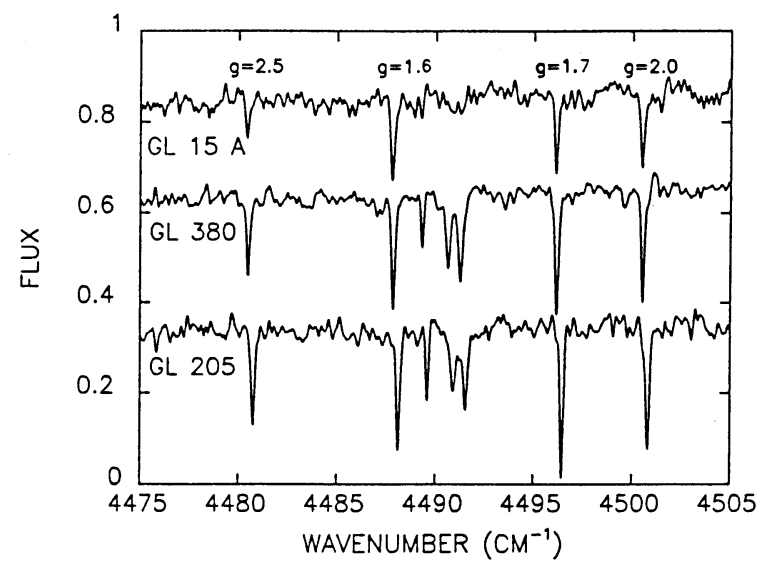

Fig. 3. Apodized 4-m FTS data for three inactive dM stars (from Saar et al. 1987). Note the lack of significant line broadening/splitting or differential shape changes in the $\mathrm{Ti} I$ multiplet indicated. 
the 4-m FTS to push even further into the IR and detect the highly Zeemansensitive $12 \mu \mathrm{m} \mathrm{Mg}$ I lines in stars. As the instrument was only able to observe very bright, magnetically inactive stars ( $\alpha$ Ori and $\alpha$ Tau), no field detections were made. Nevertheless, the lines are clearly worthy of further study once improved instruments are available, since they afford exceptional Zeeman sensitivity and a unique view of $B$ in the upper photospheres of stars (e.g., Deming et al. 1988; Solanki 1993).

IR lines were also used in passing by Saar (1988a) to demonstrate that $\epsilon$ Eri had relatively low magnetic flux (unlike occasional high values obtained earlier using non-RT methods). Basri and Marcy (1988), however, noted that the $2.2 \mu \mathrm{m} \mathrm{Na}$ I line modeled was quite optically thick and could actually be fit with a low $f$ field using a more realistic model atmosphere. More sophisticated modeling methods generally agree on $B=1-2 \mathrm{kG}$ and $f=0.15-0.30$ for $\epsilon$ Eri (Marcy and Basri 1989; Saar 1990) with remaining differences probably explained by the difference in the heights of formation of various lines (Grossmann-Doerth and Solanki 1990).

More recently, an analysis of several co-added K band spectra of AD Leo showed anomalous broadening in the $\sigma$ components of the Ti I lines (Fig. 4; Saar 1992a). Most non-magnetic broadening mechanisms could be eliminated: The lines are weak (ruling out opacity broadening), $v \sin i$ is low $\left(\sim 5 \mathrm{~km} \mathrm{~s}^{-1}\right)$, and $>10 \mathrm{~km} \mathrm{~s}^{-1}$ turbulence in magnetic regions seems implausible. Saar (1992a) suggested that the extra broadening as due to either a distribution of regions with differing $B$ on the surface (e.g., spot and plage) or a vertical gradient in $B$ over the level of line formation. The first option suggests the possible separation of weak (network/plage) and strong (umbral) fields. The latter possibility is also interesting, as it permits the line to be fit with considerably smaller $f$ values ( $~ 50 \%$ lower) than traditional analyses. It is also consistent with the prediction of lowered canopy heights in the cooler, denser atmospheres of these stars (Solanki and Steiner 1990).

AD Leo was once more the focus of study in May 1991, when a large multiwavelength campaign was launched to monitor the star's activity, including IUE, HST, ROSAT, Ginga, radio, sub-mm, IR and optical measurements (Saar et al. 1991; Bookbinder, Walter, and Brown 1992). Five nights of IR FTS spectra taken during the campaign were combined to yield spectra at three rotational phases. Systematic shape changes visible in the Ti I line profiles suggested varying magnetic region geometry and/or coverage (Fig. 5). Approximate models of the Ti I lines with a vertical $B$ gradient (assuming a homogeneous field distribution) supported this first impression, yielding tentative evidence for phase-modulated magnetic structures. We are continuing to analyze this large data set.

Finally, Saar (1992b) has made new measurements (and remeasurements using improved methods) of several KM dwarfs and RS CVn stars. These analyses have been made with an improved version of the Saar (1988a) code, which includes magneto-optical effects and a full disk-integration of the Stokes parameters (Saar et al. 1990). The improved models lower the derived filling factors somewhat, but $f$ on most flare stars remains high $(\geq 40 \%)$. The analyses of RS CVn stars make use of the increase in equivalent width of optically thick lines in a magnetic field (see Leroy 1962; Basri, Marcy, and Valenti 1992), and are less precise; only the 

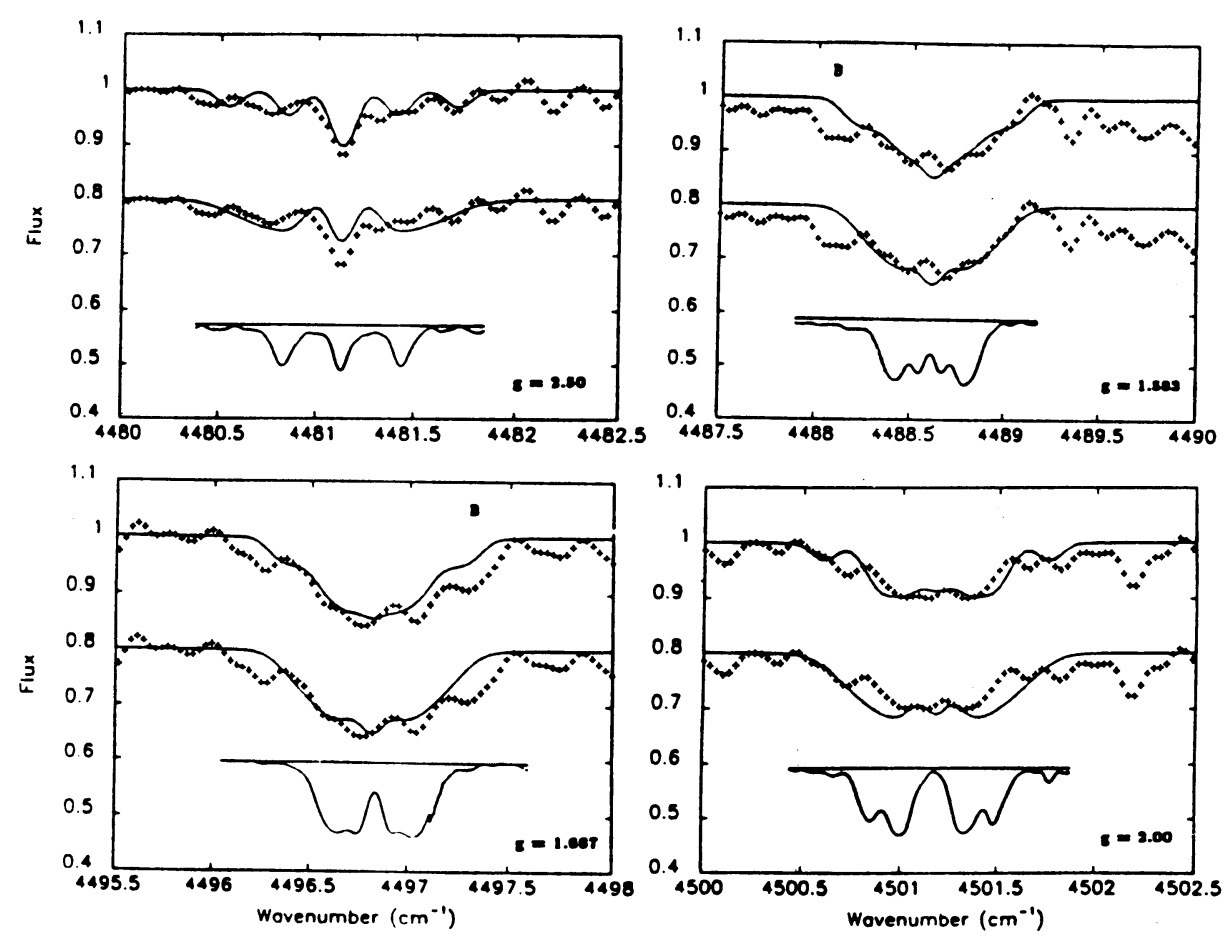

Fig. 4. Apodized, co-added Ti I line profiles from AD Leo (+) and a sunspot/quiet-Sun ratio spectrum (heavy solid; from Saar 1992a). The AD Leo lines have different shapes and appear more "blurred" than the umbral features (see text). Curves through the AD Leo spectra show best fits for two-component distributed field models (top; $f_{1}=0.45$, $\left.B_{1}=2.4 \mathrm{kG}, f_{2}=0.30, B_{2}=5.0 \mathrm{kG}\right)$ and an approximate vertical gradient model with $f=0.45, \bar{B}=3.0 \mathrm{kG}$, and $\sigma_{B}=1.6 \mathrm{kG}$.

product $f B$ is reasonably well determined. I refer the reader to Saar $(1992 b)$ for more details.

\section{IR Measurements in Context: What Have We Learned?}

The IR magnetic field measurements described above, while few in number, have played an important role in shaping the understanding of magnetic fields in cool stars. In this Section I combine these data with new IR $f \cdot B$ data (Saar 1993), some results from Saar et al. (1987) not in the Saar (1990) list, and the best optical determinations (compiled by Saar 1990, 1991); I then use these data to briefly explore the relationships between $f, B$, and other stellar parameters, and to show the role of IR data in defining them.

With detections of RS CVns at low $B$ and $M$ dwarfs at high $B$, IR data have been 


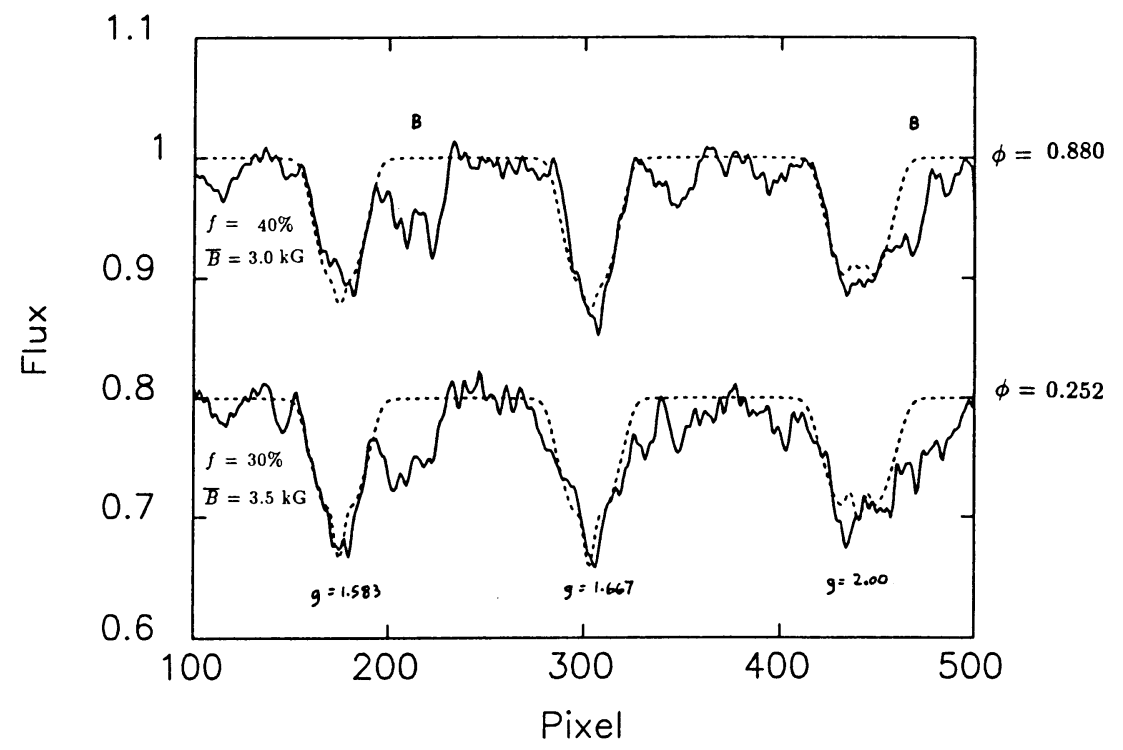

Fig. 5. Unapodized, smoothed profiles $\left(4,488,4,496,4,501 \mathrm{~cm}^{-1} ; 0.16 \AA /\right.$ pixel $)$ from AD Leo at two rotational phases, $\phi$. The lines have been "spliced together" for convenient display. The profiles appear to have systematically different shapes at the two phases, suggesting different magnetic field structures. Rough fits using an approximate $\nabla B$ model (Saar 1992a), yield the indicated $\bar{B}$ and $f$ values.

instrumental in greatly extending the range of magnetic field strengths observed in cool stars. This, in turn, has strengthened the initial indications (Saar and Linsky 1986 ) that $B$ is limited by photospheric gas pressure. Figure 6 shows the observed $B$ for the combined data set plotted against $B_{\text {eq }}=\left(\mathrm{P}_{\text {gas }, *} / \mathrm{P}_{\text {gas }, \odot}\right)^{0.5} \times B_{\odot}$, where $\mathrm{P}_{\text {gas }}$ is taken from an atmospheric model at $\tau_{5000}=1$, and $B_{\odot} \equiv 1.5 \mathrm{kG}$ (e.g., Saar 1990). For the most part, $B \leq B_{\text {eq }}$, suggesting that the external $\mathrm{P}_{\text {gas }}$ plays an important role in confining $B$ in stellar photospheres (outside of spots).

Most of the dMe flare stars are rapid rotators, and thus IR data are also important for defining the relation between $f$ and $f B$ and rotation (Saar and Linsky 1986; Marcy and Basri 1989: Saar 1990,1991). Figure 7 shows the correlation between $f$ and inverse Rossby number, $\tau_{C} \Omega$ (where $\tau_{C}$ is the convective turnover time, and I take $\Omega=\mathrm{P}_{\text {rot }}^{-1}$ ). The IR data are especially helpful for $\tau_{C} \Omega \gtrsim 0.75$, where $f$ appears to saturate. Below this value, $f \propto\left(\tau_{C} \Omega\right)^{\alpha}$, where $1.25 \lesssim \alpha \lesssim 2.0$, depending on the weight given the solar point. This relationship is somewhat steeper than determined before (e.g., Saar 1991), largely because here I have taken $f_{\odot}=0.01$ (which is probably more realistic; e.g., Solanki 1993). The correlation is consistent with the rotational evolution models of MacGregor and Brenner (1991) and with several activity theories (e.g., Jordan 1991). One can also construct $\tau_{C} \Omega$ vs. $f B, \Omega$ vs. $f$ and $f B$ diagrams, with similar results (Saar 1990; 1991). Thus, the magnetic filling factor, $f$, is the primary quantity which varies as a star evolves and slowly 


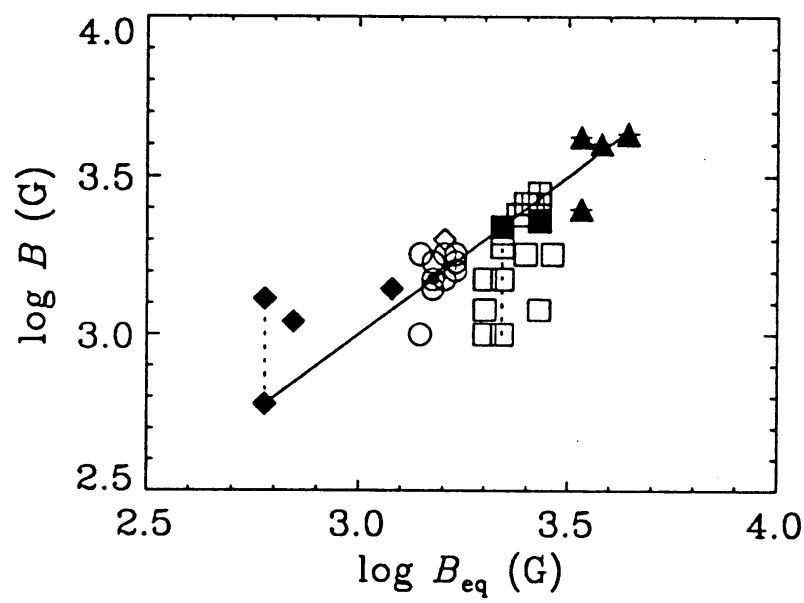

Fig. 6. Measured $B$ vs. $B_{\text {eq }}$ : circles, squares, triangles, and diamonds are G, K, M, and low-gravity stars (RS CVn, T Tau), respectively. IR measurements are filled, the Sun is designated by $\odot$ and multiple measurements are connected by dotted lines. Most points lie near or below the $B=B_{\text {eq }}$ line.

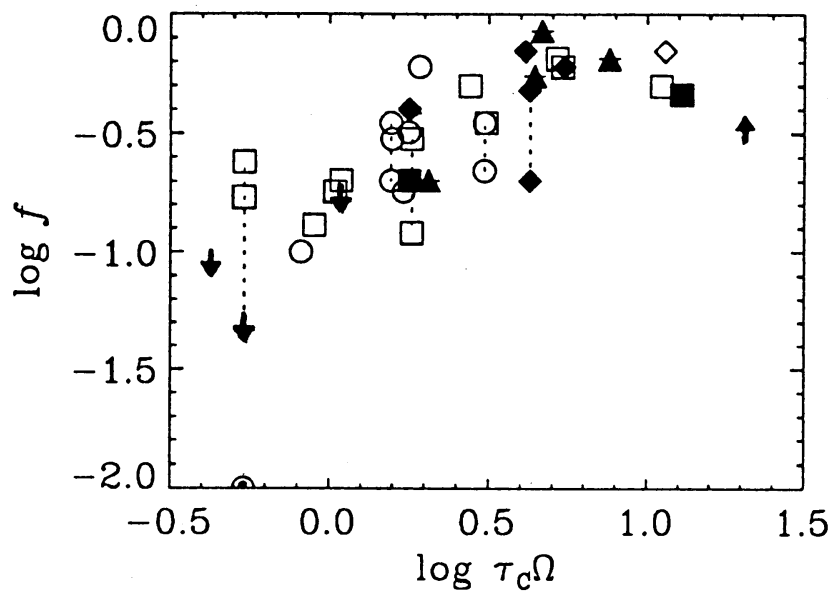

Fig. 7. Measured $f$ vs. $\tau_{C} \Omega$; symbols are asigned as in Figure 6. A positive relation can be seen for $\tau_{C} \Omega \lesssim 0.75$, after which $f$ appears to saturate. 


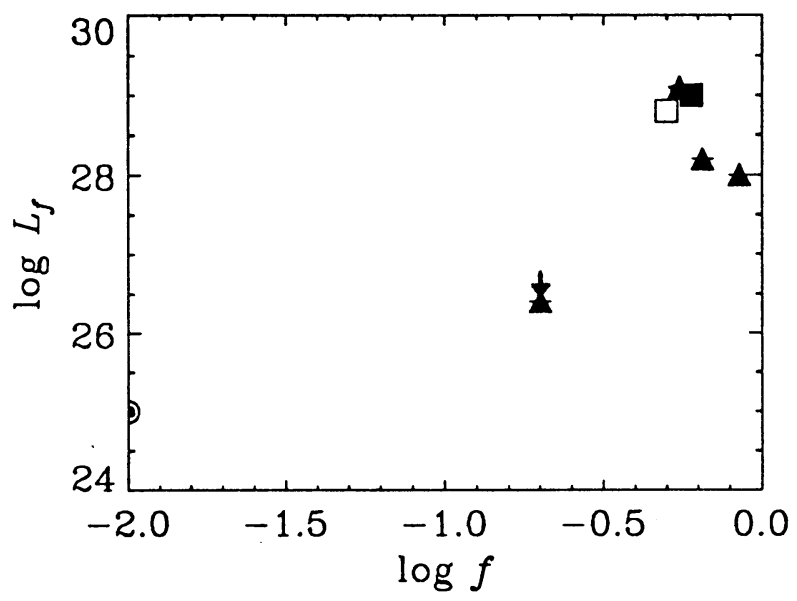

Fig. 8. Time averaged flare luminosity $L_{f}$, vs. $f$ (symbols as in Fig. 6). No clear correlation is seen.

loses angular momentum.

One must be a bit careful in the interpretation of the above results, however: Optical $f B$ measurements refer to lines formed at different depths in the stellar atmosphere than the IR features (e.g., Grossmann-Doerth and Solanki 1990). A range of formation heights (and hence $B$ ) may in fact account for much of the spread around the $B-B_{\text {eq }}$ relation, for example. On the other hand, most of the optical measurements (made near 6,170 $\AA$ ) are made at nearly the same continuum optical depth as the $2.2 \mu \mathrm{m}$ Ti I data. More realistic atmospheric and $B$ gradient models (Basri, Marcy, and Valenti 1990; Saar and Solanki 1992) are required to sort out precisely where the lines are formed. Only then can we say accurately which $B_{\text {eq }}$ should be compared to a given $B$ value, and what height $f$ refers to (although the product $f B$ will be constant throughout). There is also the question of the origin of the fields. Since the continuum contrast between spot and plage is so high in the optical, $f B$ measurements made there surely derive from plage/networklike regions. In the IR the contrast is much smaller, so IR $f$ and $B$ values may have contributions from stellar umbrae (which may have quite strong fields; Mullan 1984). The low gravity stars (RS CVns - diamonds) tend to lie somewhat above the $B=B_{\text {eq }}$ line, perhaps precisely because larger numbers of (higher $B$ ) spots on their surfaces tend to increase the measured $\bar{B}$.

There are also good correlations between magnetic flux density $f B$ and traditional signatures of magnetic "activity" in the chromosphere and corona (Saar and Schrijver 1987; Schrijver et al. 1989) and in the transition region (Saar 1988b; Schrijver 1990). The correlations, which also hold for individual active regions on the Sun, are approximately $F_{\mathrm{CHR}} \propto(f B)^{0.5}, F_{\mathrm{TR}} \propto(f B)^{0.75}$, and $F_{\mathrm{COR}} \propto(f B)^{1.0}$, where $F_{\mathrm{CHR}}, F_{\mathrm{TR}}$, and $F_{\mathrm{COR}}$ are the flux densities in the chromosphere, transition region, and corona, respectively (see also Schrijver 1991). 
Since all flare stars have large $f$ values, and magnetic reconnection would sensibly increase with $f$, it seems reasonable to also look for correlations between, for example, integrated flaring energy and $f$. Figure 8 shows the correlation between $L_{f}$, the time averaged flare luminosity (from Doyle, Byrne, and Butler 1986; Shakhovskaya 1989; and Pettersen 1990) and $f$. There may be a weak trend with $L_{f} \propto f^{2}$, but there are, sadly, only a few points and surely no clear relationship.

Many of the ambiguities in the above results should be resolved in the near future by the application of improved models to large amounts of new data from IR echelles and array detectors. IR measurements of stellar magnetic parameters will then become as important as they already are in solar physics, providing crucial information on the true field strengths, filling factors, horizontal and vertical distribution and geometry, thermodynamics, and MHD properties of stellar magnetic regions.

\section{Acknowledgements}

This work is based on data obtained at NOAO. I thank G. Basri, M. Giampapa, J. Linsky, G. Marcy, and S. Solanki for enlightening discussions. This research is supported by the NASA grant NAGW-112, Interagency Transfer W-15130, and NSF grant INT-8900202.

\section{References}

Basri, G.S., Marcy, G.W., Valenti, J.: 1992, A strophys. J. 390, 622.

Basri, G.S., Marcy, G.W., Valenti, J.: 1990, A strophys. J. 360, 650.

Basri, G.S., Marcy, G.W.: 1988, Astrophys. J. 330, 274.

Bookbinder, J.A., Walter, F.W., Brown, A.: 1992, in M. Giampapa and J. Bookbinder (eds.), Cool Stars, Stellar Systems, and the Sun, ASP Conf. Ser. 26, p. 27.

Deming, D., Boyle, R.J., Jennings, D.E., Wiedemann, G.: 1988, Astrophys. J. $333,978$.

Doyle, J.G., Byme, P.B., Butler, C.J.: 1986, Astron. Astrophys. 156, 283.

Giampapa, M.S. 1985, Astrophys. J. 299, 781.

Giampapa, M.S., Worden, S.P.: 1982, in J.O. Stenflo (ed.), Solar and Stellar Magnetic Fields: Origins and Coronal Effects, Reidel, Dordrecht, p. 29.

Giampapa, M.S., Golub, L., Worden, S.P.: 1983, Astrophys. J. (Letters) $268,121$.

Gondoin, Ph., Giampapa, M.S., Bookbinder, J.A.: 1985, A strophys. J. 297, 710.

Gray, D.F.: 1984, Astrophys. J. 277, 640.

Grossmann-Doerth, U., Solanki, S.K.: 1990, Astron. Astrophys. 238, 279.

Hall, D.: 1973, An Atlas of IR Spectra of the Solar Photosphere and Umbrae, Kitt Peak National Observatory, Tucson, Arizona.

Hall, D.N.B., Ridgway, S., Bell, E.A., Yarborough, J.M. 1979, S.P.I.E., $172,121$.

Jordan, C.J.: 1991, in P. Ulmschneider, E.R. Priest and R. Rosner (eds.), Mechanisms of Chromospheric and Coronal Heating, Springer, Berlin, p. 300.

Jennings, D.E., Deming, D., Wiedemann, G., Keady, J.J.: 1986, Astrophys. J. (Letters) 310 , L39.

Leroy, J.L.: 1962, Ann. d'Ap., 25, 127.

MacGregor, K.B., Brenner, M.: 1991, Astrophys. J. 376, 204.

Marcy, G.W., Basri, G.S.: 1989, Astrophys. J. 345, 480.

Marcy, G.W., Bruning, D.H.: 1984, Astrophys. J. 281, 286.

Mullan, D.J.: 1974, Astrophys. J. 279, 746.

Mullan, D.J.: 1975, Astron. Astrophys. 40, 41.

Pettersen, B.R.: 1990, in L.V. Miroyan, B. R. Pettersen, and M. K. Tsvetkov (eds.), Flare Stars in Star Clusters, Associations, and the Solar Vicinity, Kluwer, Dordrecht, p. 49.

Robinson, R.D.: 1980, Astrophys. J. 239, 961.

Robinson, R.D., Worden, S.P., Harvey, J.W.: 1980, Astrophys. J. (Letters) 236, L155. 
Saar, S.H.: 1992a, in M. Giampapa and J. Bookbinder (eds.), Cool Stars, Stellar Systems, and the Sun, ASP Conf. Ser. 26, p. 252.

Saar, S.H.: 1993, these proceedings.

Saar, S.H.: 1991, in I. Tuominen, D. Moss, and G. Rüdiger (eds.), The Sun and Cool Stars: Activity, Magnetism, Dynamos, Springer, Berlin, p. 389.

Saar, S.H.: 1990, in J.O. Stenflo (ed.), The Solar Photosphere: Structure, Convection, and Magnetic Fields, Kluwer, Dordrecht, p. 427.

Saar, S.H.: 1988a, Astrophys. J. 324, 441.

Saar, S.H.: 1988b, in R. Pallavicini (ed.), Hot Thin Plasmas in Astrophysics, Kluwer, Dordrecht, p. 139.

Saar, S.H., Bookbinder, J.A., Neff, J., Bromage, G., Bastian, T.: 1991, Bull. Amer. Astron. Soc. 23, 1383.

Saar, S.H., Golub, L., Bopp, B.W., Herbst, W., Huovelin, J.: 1990, in E. Rolfe (ed.), Evolution in Astrophysics, ESA SP-130, p. 431.

Saar, S.H., Linsky, J.L., Giampapa, M.S.: 1987, in L. Delbouille and A. Monfils (eds.), 27th Liège Astrophys. Colloq., U. de Liège, Liège, p. 103.

Saar, S.H., Linsky, J.L.: 1985, A strophys. J. (Letters) 299, L47.

Saar, S.H., Linsky, J.L.: 1986, Advances in Space Physics, 6, No. 8, 235.

Saar, S.H., Schrijver, C.J.: 1987, in J. Linsky and R.E. Stencel (eds.), Cool Stars, Stellar Systems, and the Sun, Springer, New York, p. 38.

Saar, S.H., Solanki, S.K.: 1992, in M. Giampapa and J. Bookbinder (eds.), Cool Stars, Stellar Systems, and the Sun, ASP Conf. Ser. 26, p. 259.

Schrijver, C.J.: 1990, Astron. Astrophys. 234, 315.

Schrijver, C.J.: 1991, in P. Ulmschneider, E. R. Priest and R. Rosner (eds.), Mechanisms of Chromospheric and Coronal Heating, Springer, Berlin, p. 257.

Schrijver, C.J., Coté, J., Zwaan, C., Saar, S.H.: 1989, A strophys. J. 337, 964.

Shakhovskaya, N.I.: 1989, Solar Phys. 121, 375.

Solanki, S.K.: 1993, these proceedings.

Solanki, S.K., Biémont, E., Mürset, U.: 1990, Astron. Astrophys. Suppl. 83, 307.

Solanki, S.K., Steiner, O.: 1990, Astron. Astrophys. 234, 519.

Strassmeier, K.G., Hall, D. S., Zeilik, M., Nelson, E., Eker, Z., and Fekel, F. C.: 1988, Astron. Astrophys. Suppl. 72, 291.

Sun, W.-H., Giampapa, M.S., Worden, S.P.: 1987 Astrophys. J. 312, 930.

Unno, W.: 1956, Pub. Astron. Soc. Japan 8, 108. 